\title{
Implicit Boundary Integral Method for Homogeneous Hele-Shaw Problem with multi-connected Domain
}

\author{
Irma Palupi \#1 \\ \# School of Computing, Telkom University \\ Bandung, Indonesia \\ ${ }^{1}$ irmapalupi@telkomuniversity.ac.id
}

\begin{abstract}
In this work, the implicit boundary integral method is implented to the homogeneous HeleShaw problem with a multi-connected domain. This method based on the solution of layer potential integral for the Laplace equation. The numerical technique is easy to implement, based on the idea of averaging the parameterization near the boundary and applying the Coarea formula. This technique changes the boundary integral into the Riemann integral that is easy to compute numerically. The difficulty in the computation of hypersingular integral occurs to compute the normal velocity of free boundary. The collocation technique is applied to eliminate the hypersingular part in the integral equation. Also, the numerical results are shown and its computation performance due to the appearance of a non-invertible matrix. In result, this method is a new technique for such a boundary problem with implementing level set method.
\end{abstract}

Keywords: Free boundary problem, Hele-Shaw problem, boundary integral method, level set method.

\begin{abstract}
Abstrak
Pada artikel ini dijelaskan implementasi metode integral permukaan secara implicit untuk permasalahan Hele-Shaw dengan domain tidak terhubung. Metode ini dikembangkan dari konsep solusi untuk persamaan Laplace dalam bentuk integral layer potensial. Dalam metode ini, teknik numerik cukup mudah dilakukan, dikarenakan bentuk integral permukaan dirubah menjadi integral Riemann melalui teknik rataan parameter dan rumus Coarea. Kendala dalam menghitung intergal tak tentu dalam rumus normal velocity dilakukan dengan cara mengeliminasi hypersigularitas dengan teknik collocation. Terakhir, ditampilkan juga beberapa numerik dan kondisi matriks selama komputasi berlangsung.
\end{abstract}

Kata Kunci: Free boundary problem, Hele-Shaw problem, boundary integral method, level set method.

\section{INTRODUCTION}

\section{A. Motivation}

I

$\mathrm{T}$ is well known for the finite difference method (FDM) and finite element method (FEM) are usually used to solve a partial differential equation numerically. In this article, the boundary integral is considered to solve our problem, which is the equation for the evolution of the boundary equation. This method works based on the integral equation in the potential theory of elliptic problem. In HaleShaw problem, as seen in (8) free boundary problem appears in solving the elliptic equation. The function 
$u$ is defined in the time depended domain $\Omega_{t}$, where its boundary evolving caused by the gradient of $u$ and some positive function $g$. Computationally, to solve the equation at time $t$, the domain at time $t$ must be known. FDM is simply to be implemented, but computationally it is not efficient for moving boundary, since the domain mesh grid is not fixed in time. It happens as well in FEM; this method also depends on constructing the mesh grid for a given domain. If the given domain change in time, then in implementing FEM it is necessary to reconstruct the mesh grid in every time step. It causes to a higher cost of computation time. Boundary integral method (BIM), considers only the change near the boundary. Furthermore, the solution is constructed in the term boundary integral by finding a density defined on the boundary by fitting a given boundary condition. These ideas relate to surface potential integral can be read further in [4], [3], [1]. In [5], they propose an implicit way to present the boundary integral equation using Coarea formula, such that the surface integral turn into Riemann integral equipped with its signeddistance representation. An important advantage of implementing this method that explains in [5] is this method is reliable for any arbitrary domain. This sustains a necessity for moving boundary problem with general velocity movement. Hele-Shaw problem is known as a popular model of incompressible liquid's flow in between two parallel plates called Hele-Shaw cell. Such a cell firstly was introduced by Henry Selby Hele-shaw as a practical model shown in his class to model a pressure driven flow of the fluid is injected into a shallow geometry from the center of two parallel plates when the surface of the fluid is bounded by another different viscous fluid or the air. The Hele-Shaw cell concept is also implemented to model dendritic form in crystalization, which has been studied following the growth pattern of SafmanTaylor in Hele-Shaw cell [7]. Also, this model is used in the application of the surface evolution between viscous fluid and melting crystal, for instance, solidification or melting process. In three dimensions, this model is used as a model of the porous medium problem, for instance the flow of water absorption inside of the sand. In the industrial application, it is used in the plastic molding, importantly to determine the way out for the melting plastic when it is filling the mold, which is the boundary between the melting plastic and the air. For the detail explanation how this model works for plastic molding and petroleum extraction, see [6].

\section{B. Hele Shaw Problem}

In this work, we perform Hele-Shaw problem in two dimensions, and the type of fluid we are working on is Newtonian viscous fluid. The pressure driven flow is caused by a given source with constant pressure in the middle of the parallel plates. The pressure driven flow in such phenomena is following the Darcy's Law that is a flow rate through the porous medium is proportional to the minus of pressure gradient of viscous fluid. Accurately, it also depends on the viscosity of the fluid and permeability of the media, but we assume they are constant for simplicity, such that from conservation law of mass, it is obtained the equation for the density changing in time as follows,

$$
\rho_{t}-\nabla \cdot \bar{q}=0
$$

where $\rho$ denotes the density over the unit volume, and $u$ is a pressure. Since we assume the fluid is incompressible, then the pressure satisfies only the Laplace equation. The governing equation can be derived from the three dimensions Navier-stock equation by averaging the gap. Let $\Omega_{0}$ be a bounded open domain. We assume that in time $t$ the fluid is filling the domain $\Omega_{t} \in \mathbb{R}^{2}$, and the boundary $\partial \Omega_{t}$ is a boundary between the liquid and the air. When the injected fluid evolves slow enough and the height $h$ of the plates is also small, the averaged velocity $\bar{q}$ over of gap satisfying

$$
\bar{q}=-\frac{h^{2}}{12 \mu} \nabla u \text {. }
$$

Since we assume the permeability and the viscosity to be constant, without losing a generality, the averaged of velocity proportional to the negative of pressure gradient, such that $\bar{q}=-\nabla u$. Therefore, the flow is almost stationary in between the gap of the plates.

$$
\Delta u=0 \quad \text { in } \Omega_{t} \backslash K,
$$

where $K$ is the area inside of $\Omega_{t}$ that is injected by the water continuously. There are two boundary conditions in the moving boundary $\Omega_{t}$, the kinematic and the dynamic boundary condition. Since this 
work is neglecting the surface tension, the dynamic boundary condition is given to be,

$$
u=0 \quad \text { on } \partial \Omega_{t}
$$

For the kinematic boundary condition, the fluid remains on the boundary in every time $t$ with normal velocity $V_{n}$,

$$
V_{n}=\bar{q} \cdot n
$$

where $n$ is the outer normal velocity. Since the pressure in $\left(\Omega_{t}\right)^{c}$ is set to be zero (the water pressure in the air), $u$ can be understood as a level set, such that

$$
\begin{aligned}
V_{n} & =\frac{u_{t}}{|\nabla u|}=-\nabla u \cdot \frac{\nabla u}{|\nabla u|} \\
\Rightarrow u_{t} & =|\nabla u|^{2}
\end{aligned}
$$

Such a problem is called homogeneous Hele-Shaw problem with the media representation is given as a constant, that is homogeneous all over the surface of the plates. Furthermore, instead of putting the media as a constant, it modifies to be a positive continuous function depends on position and time, let us denote as a function $g(x, t)$, where $\frac{1}{g(x, t)}$ can be understood as a depth of the hole must be filled by the fluid when the domain is evolving. Let $K$ be a compact set with a smooth boundary satisfying $\Omega_{0} \supset K$, where $\Omega_{0}$ is an open domain in $\mathbb{R}^{n}$ with also smooth boundary.

$$
\begin{cases}\Delta u(x, t)=0 & \text { for }(x, t) \in\left(\Omega_{t} \backslash K\right) \times(0, \infty) \\ u_{t}=g(x, t)|\nabla u|^{2} & \text { for }(x, t) \in \partial \Omega_{t} \times(0, \infty) \\ u(x, t)=1 & \text { for }(x, t) \in K \times(0, \infty) \\ u(x, t)=0 & \text { for }(x, t) \in \overline{\left(\mathbb{R}^{n} \backslash \Omega_{t}\right)} \times(0, \infty)\end{cases}
$$

For given a positive continuous function $g(x, t)$, closed set $K$, and an initial domain $\Omega_{0} \supset K$, the HeleShaw problem is reformulated as the problem of finding the pair solution of $\left(u, \Omega_{t}\right)$ satisfying (8), with initial condition $u(., 0)$ satisfying the Laplace equation in $\left(\Omega_{0} \backslash K\right)$ with suitable boundary data as in (8).

\section{Level Set Method And Numerical Algorithm}

Fast sweeping method is implemented in order to determine a level set function. Given a closed hypersurface $\Gamma$, it is evolved by giving only the velocity in the normal direction. A boundary $\Gamma$ is defined through level set representation as a zero level set of level set function $F$, i.e for each point $y \in \Gamma$, it satisfies $F(y(t), t)=0$. Thus, the level set evolution is following equation (9).

$$
\begin{aligned}
\frac{d F(y(t), t)}{d t}=0 & \\
& \Rightarrow F_{t}+\nabla F \cdot y^{\prime}(t)=0 \\
& \Rightarrow \frac{F_{t}}{|\nabla F|}+\frac{\nabla F}{|\nabla F|} \cdot y^{\prime}(t)=\frac{F_{t}}{|\nabla F|}-n_{y} \cdot y^{\prime}(t)=\frac{F_{t}}{|\nabla F|}+V_{n}=0
\end{aligned}
$$

where $V_{n}:=-n_{y} \cdot y^{\prime}(t)$ is denoted as a normal velocity.

\section{A. Initialization Technique}

In the level set representation, $\partial \Omega:\{y: F(y)=0\}$, and its normal vector $n_{y}$ is defined as

$$
n_{y}:=\frac{-\nabla F(y)}{|\nabla F(y)|}
$$

Therefore, respectively for the interior and exterior closest point $x \notin \partial \Omega$ the distance to the boundary can be computed exactly as finding $d>0$, such that $F\left(x \pm d \frac{\nabla F(x)}{|\nabla F(x)|}\right)=0$. Particular example for $\partial \Omega \subset \mathbb{R}^{2}$ and level set function $F(x)=x_{1}^{2}+x_{1}^{2}-C$, the distance of $x$ is computed by using

$$
d=\frac{(x \cdot \bar{n}(x)) \pm \sqrt{(x \cdot \bar{n}(x))^{2}-\|\bar{n}(x)\|^{2} F(x)}}{\|\bar{n}(x)\|^{2}},
$$



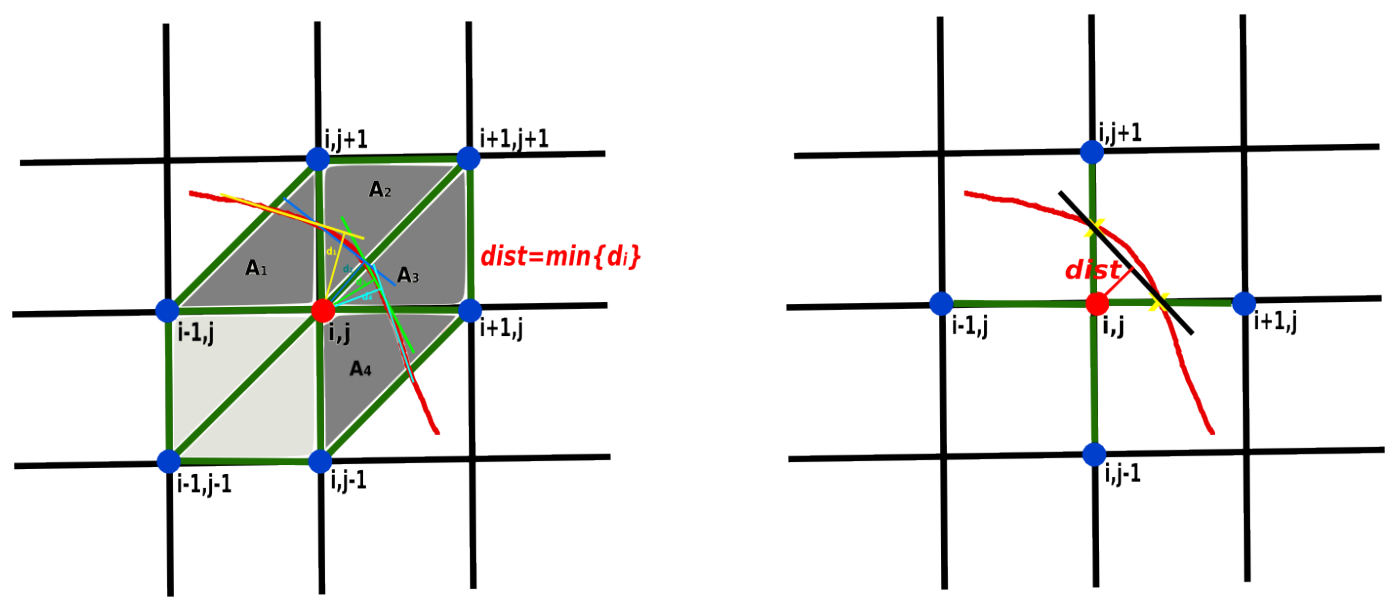

Figure 1: Ilustration how to initialize a distance function at node $(i, j)$, in case the boundary intersects two points of the node's neighborhood edges.

where $\bar{n}(x)=\frac{-\nabla F(x)}{|\nabla F(x)|}$.

If it is given only a level set data, the interpolation to compute the distance of the closest points to the boundary may be needed, since the position of the boundary curve does not intersect the grid points. Figure.1 describes the idea how to interpolate the distance at node in 2-D rectangular mesh grid. This simple idea can be used in a case the curve is nice enough being in $C^{1}(\partial \Omega)$. Given a level set data $F\left(x_{i, j}\right)$ on the meshgrid, it assumes the function $F$ is piecewise linear function in the domain, such that if the multiplication of $F\left(x_{i, j}\right)$ and the level set in its neighbors points returns negative, then the zero level set $\{x \mid F(x)=0\}$ exists along the edge connecting $x_{i, j}$ to its neighbors. Then, the distance of $x_{i, j}$ to the boundary is computed as

$$
d\left(x_{i, j}\right)=\min _{k=1,2, \ldots, 6}\left\{d_{k}\right\},
$$

where $k$ indicates the surrounding points on the hexagon in Figure. 1 with $x_{i, j}$ as a center.

\section{B. Fast Sweeping Algorithm}

The notion of Fast Sweeping algorithm based on Godunov upwind difference scheme to solve Eikonal equation. A detail description about this method can be found in [9]. In the case of distance function, a viscosity solution $d(x) \geq 0$ and it satisfies the following Eikonal equation.

$$
\left\{\begin{array}{l}
|\nabla d(x)|=1, \text { for } x \in \mathbb{R}^{n} \\
d(x)=0, \text { for } x \in \partial \Omega \subset \mathbb{R}^{n} .
\end{array}\right.
$$

Problem (12) is discretize using Godunov upwind difference scheme, such that partial derivative of $d(x)$, for $x=\left(x_{1}, x_{2}, \ldots, x_{n}\right) \in \mathbb{R}^{n}$ is approximated to be

$$
d_{x_{i}}(x)=\max \left\{\frac{d(x)-\min \left\{d\left(x+e_{i} h\right), d\left(x-e_{i} h\right)\right\}}{h}, 0\right\} .
$$

Therefore, for $n=2, \forall x$ the discrete form for (12) can be written as

$$
\max \left\{d_{i, j}-\bar{d}_{i}, 0\right\}^{2}+\max \left\{d_{i, j}-\bar{d}_{j}, 0\right\}^{2}=h^{2}
$$

where, $\bar{d}_{i}=\min \left\{d_{i+1, j}, d_{i-1, j}\right\}$ and $\bar{d}_{j}=\min \left\{d_{i, j+1}, d_{i, j-1}\right\}$.

By applying a periodic boundary condition, the one-sided nodes satisfy the following situation.

$$
\max \left\{d_{i, j}-\bar{d}_{i}, 0\right\}^{2}+\max \left\{d_{i, j}-d_{i, j-1}, 0\right\}^{2}=h^{2} .
$$

Gauss-Seidel iteration to sweep the whole distance value on the meshgrid. 
Table I: Error comparison Fast Sweeping computation performance between wo types of Initialization.

\begin{tabular}{lll}
\hline $\begin{array}{l}\text { Size of neighbor- } \\
\text { hood } \Gamma\end{array}$ & $\begin{array}{l}\text { Order of Accuracy for Initialization } \\
\text { type-1 }\end{array}$ & $\begin{array}{l}\text { Order of Accuracy for Initialization } \\
\text { type-2 }\end{array}$ \\
\hline $3 \mathrm{~h}$ & 1.75 & 1.38 \\
\hline $2 \mathrm{~h}$ & 1.85 & 1.39 \\
\hline $1.5 \mathrm{~h}$ & 1.90 & 1.42 \\
\hline $\mathrm{h}$ & 2.0 & 1.45 \\
\hline
\end{tabular}

As describe in [9], it applied the computation for rectangular grid through four alternating directions as the following ordering, such that for every $(i, j)$ do the computation as follow.

$$
\begin{array}{ll}
\text { 1). } i=0,1, \ldots, I ; j=0,1, \ldots, J & \text { 2). } i=0,1, \ldots, I ; j=J, J-1, \ldots, 1,0 \\
\text { 3). } i=I, I-1, \ldots, 1,0 ; j=J, J-1, \ldots, 1,0 & \text { 4). } i=I, I-1, \ldots, 1,0 ; j=0,1, \ldots, J
\end{array}
$$

There are several methods to compute the signed distance function after initialization. In this work uses a simple algorithm called Fast Sweeping Algorithm. The computation performance of this method depend on the accuracy in the initialization step. Table I shows the error order of Fast sweeping algorithm with two kinds of initialization as described in Figure. 1 for the circle. It concludes that the type-2 returns higher accuracy than the other.

\section{IMPLiCIT BOUNDARY INTEGRAL METHODS}

In level set method, the interface is described as a level set function in a zero level set. In a problem (9), the interface $\partial \Omega$ can be defined as $\{x \in \partial \Omega \mid F(x)=0\}$, where $F$ is a level set function. One of level set function that commonly used is a signed distance function. A signed distance function can be used to describe implicitly the interface, and it has a gradient that does not vanish around $\partial \Omega$. Moreover, it has a nice property that is $|\nabla d|=1$. Distance function is a level set function that returns the shortest distance to the nearest point on the interface. In the interface which usually can be described as a closed curve (in 2D) or closed surface, it uses a Signed distance function to sign whereas the point in interior or exterior of domain surrounded by the interface. Signed distance function respect to boundary $\partial \Omega$ is defined as follow.

$$
d(x):= \begin{cases}\inf _{y \in \Omega^{C}}|x-y| & \text { if } x \in \Omega \\ -\inf _{y \in \Omega}|x-y| & \text { if } x \in \bar{\Omega}^{c}\end{cases}
$$

One of the important properties of signed distance function if the boundary $\partial \Omega$ sufficiently smooth is, the distance function is also smooth in some tubular neighborhood of $\partial \Omega$, linear and has slope 1 along the normal direction to the interface, i.e $|\nabla d|=1$. Therefore, by using $d(x)$ as a level set function, the unit outer normal vector of $\partial \Omega$ can be obtain by the following formula.

$$
\begin{aligned}
n & =-\frac{\nabla d}{|\nabla d|} \\
& =-\nabla d
\end{aligned}
$$

According to the form of boundary integral, signed distance function is required to represent the boundary in the integral formula implicitly. The idea of the formulation is described by [5]. The notion is based on averaging the parameterization of boundary integral equation by using delta Dirac function, then use the Coarea formula, such that the boundary integral changes the form into the Riemann integral defined in $\mathbb{R}^{n}$. The signed distance function lies in the new form of integral form as we compute the value. Regularization of delta Dirac function turnout the computation consider only the points on the tubular neighborhood. See Figure. 2 for the illustration. From this state, the well known numerical integral technique can be used for computation. 


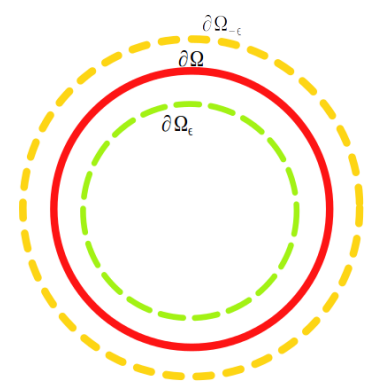

Figure 2: figure

Tubular neighborhood of $\partial \Omega$

We define a tubular neighborhood of $\partial \Omega$ with the distance not larger than $\epsilon$.

$$
\begin{aligned}
T_{\epsilon} & :=\{x \in \Omega ;|d(x)| \leq \epsilon\} \text { where, } \epsilon>0 \\
& \text { or } T_{\epsilon} \text { can be expressed as } \\
T_{\epsilon} & :=\left\{\partial \Omega_{\eta}:-\epsilon \leq \eta \leq \epsilon\right\}
\end{aligned}
$$

where $\partial \Omega_{\eta}:=\{x: d(x)=\eta\}$.

\section{A. Solution in the form of boundary integral}

For simplicity, denote $\Gamma_{t}=\partial K \cup \partial \Omega_{t}$. The solution of (8) can be constructed as a boundary integral form. We consider Double-Layer Potential (DLP) integral to construct the solution of Laplace problem in $\Omega_{t} \backslash K$, which is part of Hele-Shaw. Then, for $x \in \Omega \backslash K$ the solution of (8) is:

$$
u(x)=\int_{\Gamma_{t}} \beta(y) \frac{\partial \phi(x-y)}{\partial n_{y}} d y+A \phi\left(x, z_{0}\right), \text { for some } z_{0} \in K
$$

where $\phi$ is the fundamental solution of Laplace equation defined as the following function.

$$
\phi(x, y)= \begin{cases}-\frac{1}{2 \pi} \log |x-y| & \text { for } n=2, \\ \frac{1}{n(n-2) \rho(n)|x-y|^{n-2}} & \text { for } n \geq 3 .\end{cases}
$$

The equation (18) is fundamental solution of Laplace's equation, and for fixed $y \in \mathbb{R}^{n}$, it is a harmonic function in $\mathbb{R}^{n} \backslash\{y\}$. And the second derivative of (18) satisfies the following.

$$
\Delta \phi(x, y)=\delta_{0}(x-y) \text {. }
$$

The unknown density function $\beta$ defined on the boundary can be determined by fitting the boundary data, such that it satisfies the limit of integral DLP for $u(x)$ when $x$ approaching $\Gamma_{t}$. But the boundary integral in equation (17) is only defined in interior of domain, and to fit the density function, the limit of DLP when it is approaching the boundary is applied as in the Theorem. (1) from [4].

Theorem 1: For $\partial U$ of class $C^{2}$, the double-layer potential with continuous density $\beta$ can be continuously extended from $U$ to $\bar{U}$ and from $\bar{U}^{c}$ to $U^{c}$ with limiting values

$$
\lim _{h \rightarrow 0^{+}} \int_{\partial U} \beta(y(s)) \frac{\partial \phi\left(x \pm h n_{x}, y(s)\right)}{\partial n_{y}} d s=\int_{\partial U} \beta(y(s)) \frac{\partial \phi(x, y(s))}{\partial n_{y}} d s \mp \frac{1}{2} \beta(x)
$$

and the integral exists as an improper integral.

Therefore, according to problem (8), the density $\beta$ can be computed by solving the folowing boundary integral equation for $x \in \Gamma_{t}$.

$$
f(x)=\int_{\Gamma_{t}} \beta(y) \frac{\partial \phi(x-y)}{\partial n_{y}} d y+\frac{1}{2} \beta(x)+A \phi\left(x-z_{0}\right)
$$

where $f(x)$ is just a boundary condition written in (8),

$$
f(x)=\left\{\begin{array}{l}
0, \text { for } x \in \partial \Omega \\
1, \text { for } x \in \partial K
\end{array}\right.
$$




\section{B. Changing the Integral form}

Consider the two following integrals.

$$
\begin{gathered}
\int_{\partial \Omega} \alpha(y(s)) \psi(x, y(s)) d y(s) \\
\int_{\partial \Omega_{\eta}} \alpha(y(s)) \psi(x, y(s)) d y(s)
\end{gathered}
$$

Suppose that the arch length of a closed curve $\partial \Omega \subset \mathbb{R}^{n}$ is 1 , and let $y\left(s_{\eta}\right): s \in[0,1] \Rightarrow \partial \Omega_{\eta}$ be a parameterization on boundary $\partial \Omega_{\eta}$, then for the continuous function $f$ of our surface integral can be calculated as the following.

$$
\int_{\partial \Omega_{\eta}} f(y) d y=\int_{0}^{1} f\left(y\left(s_{\eta}\right)\right)\left|y^{\prime}\left(s_{\eta}\right)\right| d s_{\eta}
$$

We consider the integral of function that lies on $\partial \Omega$ as an integral of potential.

$$
\begin{aligned}
\int_{\partial \Omega} \alpha(y) \psi(x, y) d y & =\int_{\partial \Omega} \alpha(y) \psi(x, y) d y \\
& =\int_{\partial \Omega_{\{\eta=0\}}} \alpha(y *) \psi(x, y *) d y * \\
& =\int_{-\infty}^{\infty} \delta(\eta) \int_{\partial \Omega_{\eta}} \alpha\left(y *\left(s_{\eta}\right)\right) \psi\left(x, y *\left(s_{\eta}\right)\right) d y *\left(s_{\eta}\right) d \eta \\
& =\int_{-\infty}^{\infty} \delta(\eta) \int_{a}^{b} \alpha\left(y *\left(s_{\eta}\right)\right) \psi\left(x, y *\left(s_{\eta}\right)\right)\left|y *^{\prime}\left(s_{\eta}\right)\right| d s_{\eta} d \eta \\
& =\int_{-\infty}^{\infty} \delta(\eta) \int_{\left\{y=d^{-1}(\eta)\right\}} \alpha\left(y *\left(s_{\eta}\right)\right) \psi\left(x, y *\left(s_{\eta}\right)\right)\left|y *^{\prime}\left(s_{\eta}\right)\right| d s_{\eta} d \eta \\
& =\int_{\mathbb{R}^{n}} \delta(d(z)) \alpha(z *) \psi(x, z *)\left|y *^{\prime}\left(s_{\eta}\right)\right||\nabla d(z)| d z \text { (by using Coarea formula), } \\
& =\int_{\mathbb{R}^{n}} \delta(d(z)) \alpha(z *) \psi\left(x, z^{*}\right)\left|y *^{\prime}\left(s_{\eta}\right)\right| d z
\end{aligned}
$$

where $y *\left(s_{\eta}\right)=y\left(s_{\eta}\right)-\nabla d\left(y\left(s_{\eta}\right)\right) d\left(y\left(s_{\eta}\right)\right)$ is a projected point of $y\left(s_{\eta}\right) \in \partial \Omega_{\eta}$ to the point $y^{*}\left(s_{\eta}\right) \in \partial \Omega$. Since we use the signed distance function which $|\nabla d(y)|=1$, an outer normal vector of $\partial \Omega$ can be calculated as $n_{y} *=-\nabla d(y)$. Therefore, the formula for $\left|y *^{\prime}\left(s_{\eta}\right)\right|$ can be derived as the following formulation.

$$
\begin{aligned}
y *\left(s_{\eta}\right) & =y\left(s_{\eta}\right)-d\left(y\left(s_{\eta}\right)\right) \nabla d\left(y\left(s_{\eta}\right)\right) \\
& =y\left(s_{\eta}\right)+\eta n_{y *}\left(s_{\eta}\right)
\end{aligned}
$$

since $\tau_{s_{\eta}}=\tau\left(y\left(s_{\eta}\right)\right)=\kappa\left(s_{\eta}\right) n\left(s_{\eta}\right)$

$$
\begin{aligned}
y *\left(s_{\eta}\right) & =y\left(s_{\eta}\right)+\eta \frac{\tau_{s_{\eta}}}{\kappa\left(s_{\eta}\right)} \\
\Rightarrow y *^{\prime}\left(s_{\eta}\right) & =y\left(s_{\eta}\right)+\eta \frac{\tau^{\prime \prime}\left(s_{\eta}\right) \kappa\left(s_{\eta}\right)-\kappa^{\prime}\left(s_{\eta}\right) \tau_{s_{\eta}}}{\kappa\left(s_{\eta}\right)^{2}} \\
\Rightarrow y *^{\prime}\left(s_{\eta}\right) & =y^{\prime}\left(s_{\eta}\right)+\eta \kappa\left(s_{\eta}\right) \tau\left(s_{\eta}\right) \\
\Rightarrow y *^{\prime}\left(s_{\eta}\right) & =\left(1+\eta \kappa\left(s_{\eta}\right)\right) \tau\left(s_{\eta}\right) \quad \text { using } \tau_{s_{\eta}}=\kappa\left(s_{\eta}\right) n\left(s_{\eta}\right) \text { and } n_{s_{\eta}}=-\kappa\left(s_{\eta}\right) \tau\left(s_{\eta}\right) \\
\Rightarrow & \left|y *^{\prime}\left(s_{\eta}\right)\right|=1+\eta \kappa_{\eta}, \quad \text { if we choose } \eta<\min _{\partial \Omega_{\epsilon}} \frac{1}{\kappa_{\epsilon}(x)}
\end{aligned}
$$

Therefore, after using the chain rule, averaging the parameter with delta Dirac function, and applying the Coarea formula, now we obtain another form of DLP boundary integral (21) for a solution of Laplace 
Dirichlet boundary problem, that can be rewritten as the following statements.

\section{Double-layer potential}

1 Find density function $\beta$, such that for $x \in \partial \Omega$ it is satisfied

$$
f(x)=\int_{\mathbb{R}^{n}} \beta(y *) \frac{\partial \Phi(x, y *)}{\partial n_{y} *} \delta_{\epsilon}(y) J(y) d y-\frac{1}{2} \beta(x)
$$

where $y *$ is a projection point of $y \in \mathbb{R}^{n}$ with a formula $y *=y-d(y) \nabla d(y)$, and $J(y)$ is a Jacobian equals to $\left|y *^{\prime}\left(s_{\eta}\right)\right|$ for some parameterization $s_{\eta}$ we explained before.

2 Construct solution $u(x)$ for $x \in \Omega$ as

$$
u(x)=\int_{\mathbb{R}^{n}} \beta(y *) \frac{\partial \Phi(x, y *)}{\partial n_{y} *} \delta_{\epsilon}(y) J(y) d y
$$

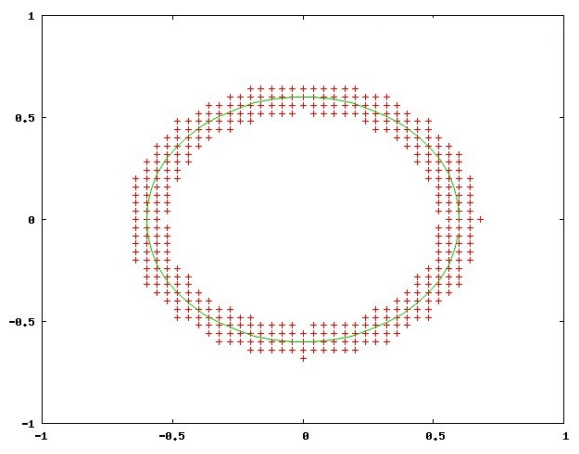

Figure 3: The closest points correspond to the circle with radius 0.6 . In this mesh example, the spacing mesh is equidistant set as $\delta x=0.04$.

\section{Algorithm of Implicit BIM for Hale-Shaw Problem}

Given an initial domain $\Omega_{0}$, the following steps are the procedure to compute the DLP in the boundary integral equation.

0 . For initial discrete time $k=0$, given a boundary $\partial \Omega^{k}$, define a level set function $F^{k}(x): \mathbb{R}^{n} \rightarrow \mathbb{R}$ such that $\partial \Omega_{0}:=\left\{x ; F^{k}(x)=0\right\}$.

1 . Initialization of distance function.

2 . Using Fast Sweeping algorithm to compute signed distance function $d_{\partial \Omega^{k}}(x)$ as explained in [5].

3 . Find the density $\beta$ to fit the boundary condition, by solving a linear system from (30).

4 . Use $\beta$ from step 3 to find the solution $u^{k}(x)$ by the formula (31). Also as the zero level set of $F^{k}$ the boundary $\partial \Omega^{k}:=\left\{x: F^{k}(x)=0\right\}$.

5 . Compute the extended of normal velocity $\bar{V}$, in the tubular neighborhood $\Gamma_{\epsilon}$. According to the DLP form $\partial u / \partial n_{x}$ defined in $V_{n}$ has the following continuous form :

$$
\frac{\partial u}{\partial n_{x}}=\int_{\partial \Omega} \beta(y) \frac{\partial^{2} \phi(x, y)}{\partial n_{y} \partial n_{x}} d y+\sum_{1}^{m t} a_{j} \frac{\partial \phi\left(x^{*}, z_{j}\right)}{\partial n_{x}}
$$

6 . Update a new level set function for the next time step $F^{(k+1)(x)}$ by computing

$$
F^{k+1}(x)=F^{k}(x)-\bar{V}(x)\left|\nabla F^{k}(x)\right| \Delta t,
$$

where $F^{k}(x)=d_{\partial \Omega^{k}}(x)$.

7 . Repeat from the step- 1 as a required time steps.

The algorithm for the procedure above described as follows. 


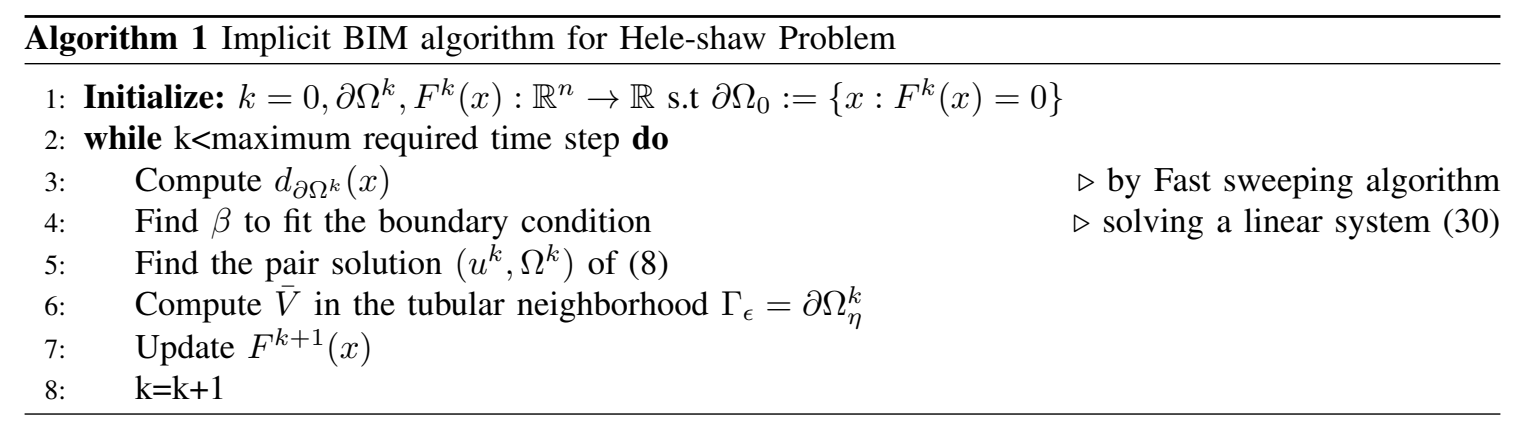

\section{DisCRETIZATION}

This section explains the discretization scheme for the integral equation on (30). The integral formula becomes easy to compute numerically as a Riemann integral. Therefore, for numeric computation, the Trapezoidal scheme is chosen for integral discretization.

\section{A. Trapezoidal Integral}

From (30) and (31), the main problem in the computation is finding a density function $\beta$ that fit to the boundary condition, then use it to construct solution $u(x)$. Remark the jacobian $J(y)$ for $y \in$ $\partial \Omega_{\eta}$ on level set $d(y)=\eta$, defined as $J(y)=1+\eta \kappa(y)$. In the level set representation by signed distance function, a curvature $\kappa=-\Delta d(y)$. Thus the Jacobian in term of Riemann integral computed as $J(y)=1-d(y) \Delta d(y)$. Therefore, for every point $x_{i}$ in the tubular neighborhood, trapezoidal integral discretization for the integral form in (30) will have the following form.

$$
f\left(x_{i}^{*}\right)=h^{n} \sum_{j=1}^{N}\left(\beta\left(y_{j}^{*}\right) \frac{\partial \phi\left(x_{i}^{*}, y_{j}^{*}\right)}{\partial n_{y}^{*}} J\left(y_{j}\right) \delta_{\epsilon}\left(y_{j}\right)\right)+\frac{1}{2} \beta\left(x_{i}^{*}\right)
$$

In order to solve (34) for $\beta$, the linear system $\left(A+\frac{1}{2} I\right) \bar{\beta}=\bar{f}$ occurs, where

$$
A_{i, j}=\frac{\partial \phi\left(x_{i}^{*}, y_{j}^{*}\right)}{\partial n_{y}^{*}} J\left(y_{j}\right) \delta_{\epsilon}\left(y_{j}\right)
$$

$\bar{\beta}=\left(\beta\left(y_{1}^{*}, \beta\left(y_{2}^{*}\right), \cdots, \beta\left(y_{N}^{*}\right)\right)\right)^{T}$, and $\bar{f}=\left(f\left(x_{1}^{*}\right), f\left(x_{2}^{*}\right), \cdots, f\left(x_{N}^{*}\right)\right)$ for $i=1,2, \ldots, N$.

In order to compute $\frac{\partial \phi\left(x_{i}^{*}, y_{j}^{*}\right)}{\partial n_{y}^{*}}=\nabla \phi \cdot n_{y}^{*}$, the normal vector $n_{y}^{*}$ equals to $-\frac{\nabla d}{|\nabla d|}$ if the signed distance function $d$ is used as a level set function. Since $|\nabla d|=1$ on the boundary, then $\frac{\partial \phi\left(x_{i}^{*}, y_{j}^{*}\right)}{\partial n_{y}^{*}}=$ $-\nabla_{y} \phi\left(x_{i}^{*}, y_{j}^{*}\right) \cdot \nabla d\left(y_{j}^{*}\right)$. However, $\nabla d$ is defined only on the grid points. We can use bi-linear interpolation to approach the value of $\nabla d$ defined on the boundary. Finally the double layer potential $u(x)$ for $x \in \Omega$ can be obtained by using the following discretization.

$$
u(x)=h^{n} \sum_{j=1}^{j=N} \beta\left(y_{j}^{*}\right) \frac{\partial \phi\left(x, y_{j}^{*}\right)}{\partial n_{y}^{*}} J\left(y_{j}^{*}\right) \delta_{\epsilon}\left(y_{j}^{*}\right) .
$$

However, the singularity appears in computing $\frac{\partial \phi\left(x, y_{j}^{*}\right)}{\partial n_{y}^{*}}$ on the boundary, when $y_{j} *$ is close enough to $x_{i}^{*}$. The regularization is required to avoid unsolvable linear system.

The regularization of $\frac{\partial \phi\left(x, y_{j}^{*}\right)}{\partial n_{y}^{*}}$ have done by relocating $x$ and $y$ in a sphere $\partial B(0, R)$ since they are close each other. Fix for $y=(0, R)$ and any point of $x$ lies on $\partial B(0, R)$ has a polar coordinate $x=(R \cos \theta, R \sin \theta)$. On $\partial B$, normal derivative of $\phi$ is rewritten as

Formula for $\frac{\partial \phi(x, y)}{\partial n_{y}}$

$$
\frac{\partial \phi(x, y)}{\partial n_{y}}= \begin{cases}\frac{1}{4 \pi} \kappa(y) & , \text { for }|x-y|<\tau \\ \frac{\partial \phi(x, y)}{\partial n_{y}} & , \text { Otherwise }\end{cases}
$$


IRMA PALUPI

IMPLICIT BOUNDARY INTEGRAL...

B. For Multi-connected Domain

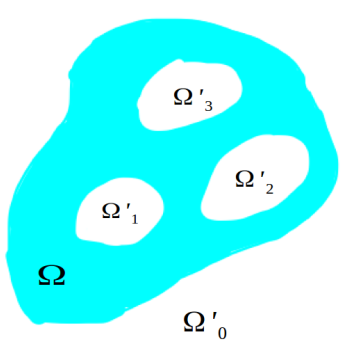

Figure 4: The example of multi-connected domain.

The DLP integral for the solution of $u(x)$ has been expressed as the integral over $T_{\epsilon} \cup \mathbb{R}^{2}$. Therefore, to fit the boundary data the following discretization we do to compute numerically the density function $\beta$. Let $\mathrm{N}$ be a number of points lie in the tubular neighborhood. For each point $x_{i}$ in the tubular neighbourhood $T_{\epsilon}$ with $x_{i}^{*}$ is its projection on the boundary, the latest of integral form can be discretized to become:

$$
f\left(x_{i}^{*}\right)=h^{n} \sum_{j=1}^{N}\left(\beta\left(y_{j}^{*}\right) \frac{\partial \phi\left(x_{i}^{*}, y_{j}^{*}\right)}{\partial n_{y}^{*}} J\left(y_{j}\right) \delta_{\epsilon}\left(y_{j}\right)\right)+\frac{1}{2} \beta\left(x_{i}^{*}\right)+A \phi\left(x_{i}^{*}, z_{k}\right)
$$

for any $z_{0} \in K$. Also, put the ekstra condition for the density such that the linear system according to (38) has non-trivial solution. And from the discretization (38), we have the linear system $A \bar{v}=\bar{F}$ to solve, where $B, \bar{v}, \bar{F}$ have the form as folows.

$$
\begin{gathered}
B=\left[\begin{array}{cc}
\left(C+\frac{1}{2} I\right) & \phi\left(\mathbf{x}_{\mathbf{i}}^{*}-\mathbf{z}_{\mathbf{0}}\right) \\
\mathbf{1} & \mathbf{0}
\end{array}\right], \text { where } C_{i, j}=h^{2} \frac{\partial \phi\left(x_{i}^{*}, y_{i}^{*}\right)}{\partial n_{y}^{*}} J\left(y_{j}\right) \delta_{\epsilon}\left(y_{j}\right) \\
\bar{v}=\left[\begin{array}{llll}
\beta\left(x_{1}^{*}\right) & \ldots & \beta\left(x_{N}^{*}\right) & A
\end{array}\right]^{t} \\
\bar{F}=\left[\begin{array}{llll}
f\left(x_{1}^{*}\right) & \ldots & f\left(x_{N}^{*}\right) & 0
\end{array}\right]^{t}
\end{gathered}
$$

\section{Extention of Normal Velocity}

To compute the normal velocity $V_{n}$ as defined before, we need to derive the formula for a normal derivative of solution $u(x)$ from the form of integral DLP.

$$
\begin{aligned}
\frac{\partial u}{\partial n_{x}}(x) & =\nabla_{x} u \cdot n_{x} \\
& =\int_{\partial \Gamma_{t}} \beta(y) \frac{\partial^{2} \phi(x-y)}{\partial n_{y} \partial n_{x}} d y+A \frac{\partial \phi\left(x-z_{0}\right)}{\partial n_{x}}
\end{aligned}
$$

The term $\frac{\partial^{2} \phi}{\partial n_{y} \partial n_{x}}$ can be written as follows.

$$
\begin{aligned}
\frac{\partial^{2} \phi}{\partial n_{y} \partial n_{x}} & =\frac{\partial}{\partial n_{y}}\left[\frac{\partial \phi(x-y)}{\partial n_{x}}\right] \\
& =\frac{\partial}{\partial n_{x}}\left[-\frac{1}{2 \pi} \frac{\left((x-y) \cdot n_{y}\right)}{|x-y|^{2}}\right] \\
& =\frac{1}{2 \pi}\left(2 \frac{\left((x-y) \cdot n_{y}\right)\left((x-y) \cdot n_{x}\right)}{|x-y|^{4}}-\frac{\left(n_{y} \cdot n_{x}\right)}{|x-y|^{2}}\right)
\end{aligned}
$$


Therefore, for $x \in \Gamma_{t}$ normal velocity $V=\left|\partial u / \partial n_{x}\right|$, where

$$
\begin{aligned}
\frac{\partial u}{\partial n_{x}} & =\frac{1}{2 \pi} \int_{\Gamma_{t}} \beta(y)\left(2 \frac{\left((x-y) \cdot n_{y}\right)\left((x-y) \cdot n_{x}\right)}{|x-y|^{4}}-\frac{\left(n_{x} \cdot n_{y}\right)}{|x-y|^{2}}\right) d y \\
& +\frac{A}{2 \pi} \frac{\left(\left(x-z_{0}\right) \cdot n_{x}\right)}{\left|x-z_{0}\right|^{2}} .
\end{aligned}
$$

For particular case i.e annulus domain, let we denote two additional terms at (47) as $T_{1}$ and $T_{2}$ are the following.

$$
\begin{gathered}
T 1=\int_{\partial(\Omega K)} \beta(y) \frac{\partial^{2} \phi}{\partial n_{x} \partial n_{y}} d y \\
T 2=A \phi\left(x-z_{0}\right), \text { for some } z_{0} \in K
\end{gathered}
$$

Then we observe $T 1$ according to the constant boundary data as given in (22).

$$
\begin{aligned}
T 1 & =\int_{\partial \Omega} \beta(y) \frac{\partial^{2} \phi}{\partial n_{x} \partial n_{y}} d y+\int_{\partial K} \beta(y) \frac{\partial^{2} \phi}{\partial n_{x} \partial n_{y}} d y \\
& =\beta_{1} \int_{\partial \Omega} \frac{\partial^{2} \phi}{\partial n_{x} \partial n_{y}} d y+\beta_{2} \int_{\partial \Omega} \frac{\partial^{2} \phi}{\partial n_{x} \partial n_{y}} d y,(\text { since } \beta \text { is constant from constant boundary data, }) \\
& \left.\left.=\beta_{1} \int_{\Omega} 1 \Delta\left(\frac{\partial \phi}{\partial n_{x}}\right)+\nabla\left(\frac{\partial \phi}{\partial n_{x}}\right) \cdot 0\right) d x+\beta_{2} \int_{K} 1 \Delta\left(\frac{\partial \phi}{\partial n_{x}}\right)+\nabla\left(\frac{\partial \phi}{\partial n_{x}}\right) \cdot 0\right) d x(\text { by integration by part) } \\
& =0
\end{aligned}
$$

Thus, for annulus domain with given constant boundary data,

$$
\frac{\partial u}{\partial n_{x}}=T 2
$$

However in general, the integral (47) appears as hypersingular integral, and in the problem (8), $\partial \Omega_{t} \subset$ $\Gamma_{t}:=\{x \mid d(x, t)=0\}$ moves with normal velocity $V_{n}$, with fixed boundary $\partial K$. Instead of computing the normal velocity on the $\partial \Omega_{t}$, we moves a curve by finding the extension of normal velocity in $T_{\epsilon}$ and update the level set function around $T_{\epsilon}$. And we notice that the extension only requires to be continous. Denote the extension of $V_{n}$ to be $\widetilde{V_{n}}(x, \tau)$ such that it is defined in $\left(T_{\epsilon} \cup \mathbb{R}^{2} \times(0, \infty)\right)$. Then, we update the new level set only in the neighbourhood of $\partial \Omega_{t}$ by using the following formula. For $x \in T_{\epsilon} \backslash \Gamma_{t}$, the formula for $\widetilde{V_{n}}=\left|\widetilde{\partial u / \partial n_{x}}\right|$ is:

$$
\begin{aligned}
& \frac{\widetilde{\partial u}}{\partial n_{x}}(x)=\frac{1}{2 \pi} \int_{\Gamma_{t}} \beta(y)\left(2 \frac{((x-y) \cdot \nabla d(y))((x-y) \cdot \nabla d(x))}{|x-y|^{4}}-\frac{(\nabla d(y) \cdot \nabla d(x))}{|x-y|^{2}}\right) d y \\
& -\frac{A}{2 \pi} \frac{\left(\left(x-z_{0}\right) \cdot \nabla d(x)\right)}{\left|x-z_{0}\right|^{2}} \text { and by Coarea formula, } \\
& =\frac{1}{2 \pi} \int_{T_{\epsilon}} \beta\left(z^{*}\right)\left(2 \frac{\left(\left(x-z^{*}\right) \cdot \nabla d\left(z^{*}\right)\right)\left(\left(x-z^{*}\right) \cdot \nabla d(x)\right)}{\left|x-z^{*}\right|^{4}}-\frac{\left(\nabla d\left(z^{*}\right) \cdot \nabla d(x)\right)}{\left|x-z^{*}\right|^{2}}\right) J(z) \delta_{\epsilon}(z) d z \\
& -\frac{A}{2 \pi} \frac{\left(\left(x-z_{0}\right) \cdot \nabla d(x)\right)}{\left|x-z_{0}\right|^{2}}
\end{aligned}
$$

To compute the last equation, we use simple Quadrature rules to compute numerical integration. The discrete from of the integral is as follows.

$$
\begin{aligned}
& \frac{\widetilde{\partial u}}{\partial n_{x}}\left(x_{i}\right) \approx \frac{1}{2 \pi} \sum_{j=1}^{j=N} h^{2} \beta\left(y_{j}^{*}\right)\left(2 \frac{\left(\left(x_{i}-y_{j}^{*}\right) \cdot \nabla d\left(y_{j}^{*}\right)\right)\left(\left(x_{i}-y_{j}^{*}\right) \cdot \nabla d\left(x_{i}\right)\right)}{\left|x_{i}-y_{j}^{*}\right|^{4}}-\frac{\left(\nabla d\left(y_{j}^{*}\right) \cdot \nabla d\left(x_{i}\right)\right)}{\left|x_{i}-y_{i}^{*}\right|}\right) J\left(y_{j}\right) \delta_{\epsilon}\left(y_{j}\right) \\
& -\frac{A}{2 \pi} \frac{\left(x_{i}-z_{0}\right) \cdot \nabla d\left(x_{i}\right)}{\left|x_{i}-z_{0}\right|^{2}}
\end{aligned}
$$




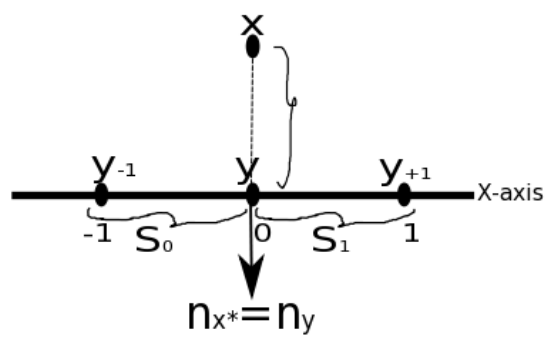

Figure 5: figure

Collocating hypersingular integral in 2D

Since (50) is hypersingular integral, we still need to regularize the value when $x \in\left(T_{\epsilon} \backslash \Gamma_{t}\right)$ is very close to some $y \in \Gamma_{t}$. The regularization for the hypersingular integral has been proposed in [8] by using the method named collocation to interpolate the value of $\beta$.

\section{Regularization for the extended Velocity Formula}

The formula of normal velocity include a hypersingular integral on the boundary $\partial \Omega_{t}$, and we agreed that to extend the velocity at the point passing through the boundary by defining a continuous extension, i.e $\tilde{V}_{n}$. However, the integral term in the formula still requires a regularization for $x$ close to boundary. One of basic approximations is collocation. Remind that in calculating the integral of double layer potential, collocation/re-coordinating is applied to regularize the occurred term $\frac{\partial \phi(x, y)}{\partial n_{y}}$ when $x$ close to $y \in \Gamma_{t}$. We describe 2D geometry for the idea of regularization the second derivative of boundary integral.

A small edge of the boundary is projected to the $\mathrm{X}$-axis such that we can consider this part as a flat boundary segment consisting the interval $[-1,1]$, where by limiting value of $\epsilon \rightarrow 0 \ldots$

To approximate the boundary and the function $\beta$ lies on the boundary, we use linear interpolation with linear shape functions are follows.

$$
\begin{array}{r}
\varphi_{1}(s)=1-s \\
\varphi_{2}(s)=s
\end{array}
$$

The linear interpolation on $S_{0}$ and $S_{1}$ for the boundary and the function $\beta$ respectively are defined as : For $\Gamma_{0}$

$$
\begin{array}{r}
\Gamma_{0}(s)=y_{-1} \varphi_{1}(s)+y_{0} \varphi_{2}(s)=(-1+s, 0) \\
\beta\left(\Gamma_{0}(s)\right)=\beta\left(y_{-1}\right) \varphi_{1}(s)+\beta\left(y_{0}\right) \varphi_{2}(s)=\beta\left(y_{-1}\right)+s\left(\beta\left(y_{0}\right)-\beta\left(y_{-1}\right)\right) \\
|x-y|^{2}=(-1+s)^{2}+\epsilon^{2}
\end{array}
$$

for $\Gamma_{1}$

$$
\begin{array}{r}
\Gamma_{1}(s)=y_{0} \varphi_{1}(s)+y_{1} \varphi_{2}(s)=(s, 0) \\
\beta\left(\Gamma_{1}(s)\right)=\beta\left(y_{0}\right) \varphi_{1}(s)+\beta\left(y_{1}\right) \varphi_{2}(s)=\beta\left(y_{0}\right)+s\left(\beta\left(y_{1}\right)-\beta\left(y_{0}\right)\right) \\
|x-y|^{2}=s^{2}+\epsilon^{2}
\end{array}
$$


The computation of the limit integral can be shown as follow.

$$
\begin{aligned}
\lim _{\epsilon \rightarrow 0^{+}} \int_{S_{0} \cup S_{1}} \beta & \beta(y) \frac{\partial^{2} \phi(x, y)}{\partial n_{x}^{*} n_{y}} d y=\lim _{\epsilon \rightarrow 0^{+}} \frac{1}{2 \pi} \int_{S_{0} \cup S_{1}} \beta(y)\left[\frac{n_{x} \cdot n_{y}}{|x-y|^{2}}-2 \frac{\left(n_{x} \cdot(x-y)\right)\left(n_{y} \cdot(x-y)\right)}{|x-y|^{4}}\right] d y \\
& =\lim _{\epsilon \rightarrow 0^{+}} \frac{1}{2 \pi} \int_{S_{0}} \beta(y)\left[\frac{n_{x} \cdot n_{y}}{|x-y|^{2}}-2 \frac{\left(n_{x} \cdot(x-y)\right)\left(n_{y} \cdot(x-y)\right)}{|x-y|^{4}}\right] d y \\
& +\lim _{\epsilon \rightarrow 0^{+}} \frac{1}{2 \pi} \int_{S_{1}} \beta(y)\left[\frac{n_{x} \cdot n_{y}}{|x-y|^{2}}-2 \frac{\left(n_{x} \cdot(x-y)\right)\left(n_{y} \cdot(x-y)\right)}{|x-y|^{4}}\right] d y \\
& =\lim _{\epsilon \rightarrow 0^{+}} \frac{1}{2 \pi} \int_{0}^{1} \beta\left(y_{-1}\right)+s\left(\beta\left(y_{0}\right)-\beta\left(y_{-1}\right)\right)\left[\frac{1}{(-1+s)^{2}+\epsilon^{2}}-\frac{2 \epsilon^{2}}{\left((-1+s)^{2}+\epsilon^{2}\right)^{2}}\right] d s \\
& +\lim _{\epsilon \rightarrow 0^{+}} \frac{1}{2 \pi} \int_{0}^{1} \beta\left(y_{0}\right)+s\left(\beta\left(y_{1}\right)-\beta\left(y_{0}\right)\right)\left[\frac{1}{s^{2}+\epsilon^{2}}-\frac{2 \epsilon^{2}}{\left(s^{2}+\epsilon^{2}\right)^{2}}\right] d s \\
& =\lim _{\epsilon \rightarrow 0^{+}} \frac{1}{2 \pi}\left[\beta\left(y_{-1}\right) \frac{-1}{1+\epsilon^{2}}+\left(\left(\beta\left(y_{0}\right)-\beta\left(y_{-1}\right)\right)-\left(\beta\left(y_{1}\right)-\beta\left(y_{0}\right)\right)\right) \log \left(\epsilon^{2}\right) / 2+\beta\left(y_{0}\right) \frac{-1}{1+\epsilon^{2}}\right]
\end{aligned}
$$

By using this approximation, the integral is finite if only if the term consist $\log \left(\epsilon^{2}\right) / 2$ canceled out, and this happens only if

$$
\beta\left(y_{0}\right)-\beta\left(y_{-1}\right)=\beta\left(y_{1}\right)-\beta\left(y_{0}\right)
$$

which we conclude that a function $\beta$ must be in $C^{1}$. Thus, from the latest computation we have :

$$
\lim _{\epsilon \rightarrow 0^{+}} \int_{S_{0} \cup S_{1}} \beta(y) \frac{\partial^{2} \phi(x, y)}{\partial n_{x}^{*} n_{y}} d y=-\frac{1}{2 \pi}\left(\beta\left(y_{-1}\right)+\beta\left(y_{0}\right)\right)
$$

\section{REsults AND Discussion}

In the discretization of singular integral as we have in DLP boundary integral, where the integral over the surface is transformed into the integral defined in $\mathbb{R}^{n}$, it is important to approximate the delta Dirac function. We use one of two techniques that was proposed by [2], i.e variable regularization parameter. In this computation, we use the following averaging kernel for delta Dirac function in the formula.

$$
\left\{\begin{array}{l}
\delta_{\epsilon}(x)=\frac{1}{2 \epsilon}\left(1+\cos \left(\frac{\pi x}{\epsilon}\right)\right) \\
0 \quad \text { otherwise }
\end{array}\right.
$$

The convergence of $\beta$ as the grid increases was experimented by the work of [5] inspired by the result of [2] about the convergence of surface integrals in the Cartesian grid. So we choose the same tubular neighborhood size around the boundary as in [5],

$$
\epsilon=2|\nabla d|_{1} h
$$

where $h$ is a mesh size of equidistant discretization in the Cartesian grid. Figure. 6 shows the numerical solution of Hele-Shaw problem defined in (8). The algorithm in Section III-C is applied to the simply connected domain with only one connected source $K \subset \Omega_{t}$ and to the multi-connected ellipse with disconnected source $K$. Since finding the $\beta$ requires to solve the integral equation that contains the singular integral and the regularization causes the ill-conditioned matrix during the computation (noninvertible coefficient matrix in (34)), the singular decomposition value technique is used to obtain the solution. The matrix condition number can be seen in Figure. (7).

Figure. (7) shows ill-conditioned matrix occurs very large during computation to find the coefficient $\beta$. The coeficient matrix of linear system (34) is not invertible, such that problem (34) in this work is solved by using singular decomposition value (SDV). Surprisingly, the larger matrix condition value does not depend on the mesh size $N$. The condition matrix of mesh size $N=960$ appears to be better that $N=600$. 


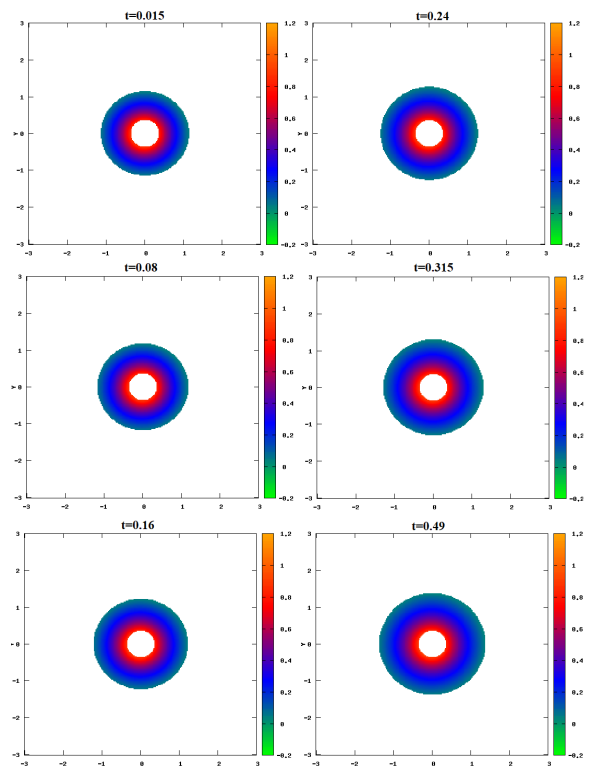

(a)

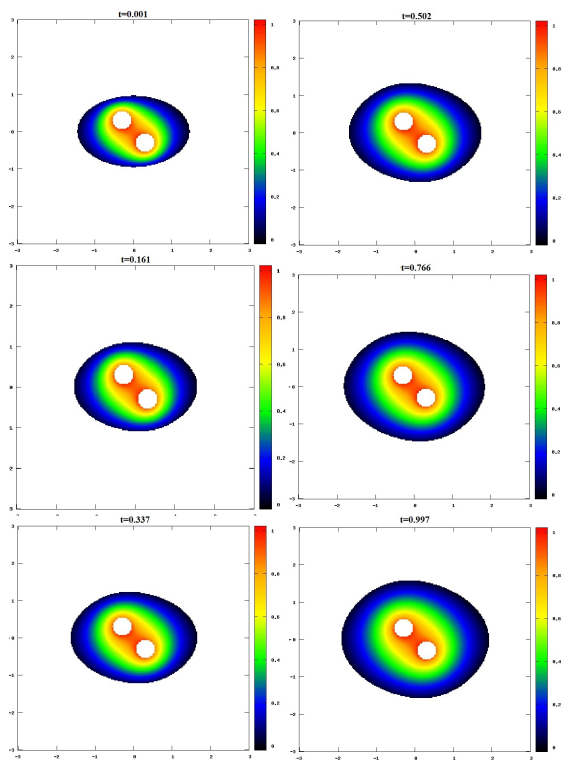

(b)

Figure 6: Performs the evolution of the domain in the numerical solution of Hele-shaw problem by using implicit BIM. (a) shows the evolution of the boundary with the given initial domain $\Omega_{0}=B\left(0, r_{1}\right)$ as a circle with only one the injection source $K=B\left(0, r_{2}\right)$ for $r_{1}>r_{2}>0 . B\left(0, r_{1}\right) \backslash B\left(0, r_{2}\right)$ is a simple-connected domain. Figure (b) shows the evolution of the domain as multi-connected ellipse with two disconnected injecting area inside of it.

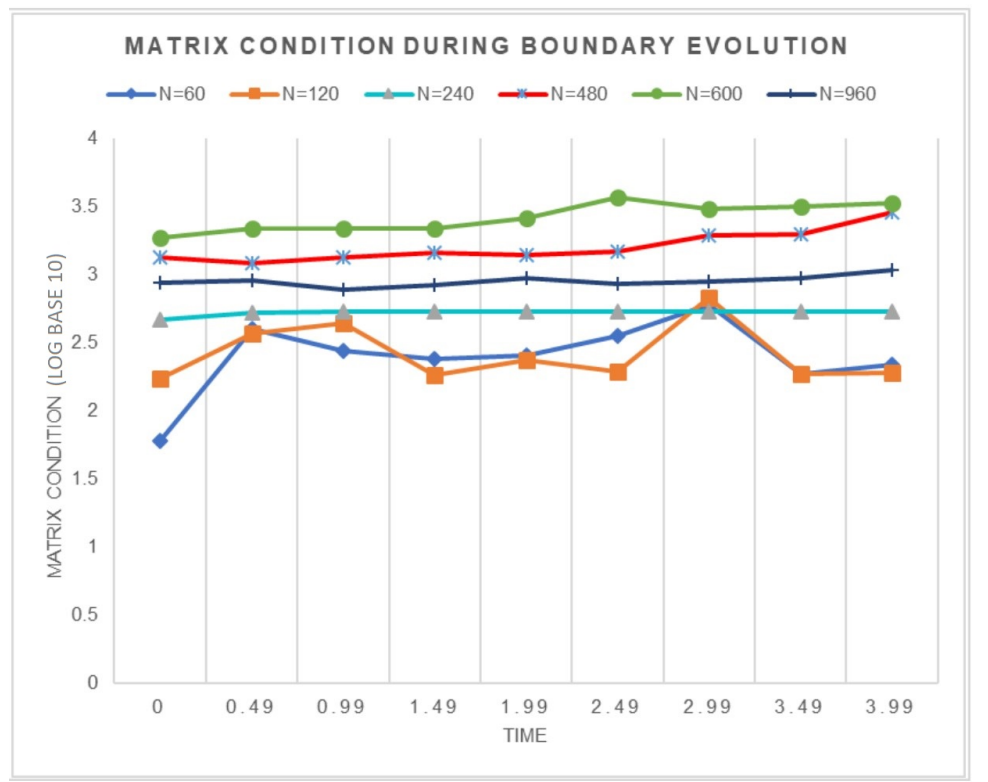

Figure 7: Matrix condition during the domain evolution.

\section{CONCLUSION}

This work implements the method names implicit boundary integral method to approximate the Laplacian in two dimension that included in the Hele-Shaw problem, and naively compute the normal velocity to update the free boundary. The algorithm based on the solution of the boundary integral equation is not only for the simply-connected domain but also for the domain that has a disconnected sources. Since in 
this method the boundary is described as a level set function and is also grid independent, this method has a benefit for such a free boundary problem as considered in this work. The improvement is also necessary to increase the accuracy and the efficiency in the computation technique, specially in handling the ill-conditioned matrix that exist during computation.

\section{ACKNOWLEDGMENT}

The author would like to thank to Norbert Pozar for introducing this topic two years ago. Also, the author thank to School of Computing, Telkom University for all of the given supports.

\section{REFERENCES}

[1] David Colton and Rainer Kress. Integral equation methods in scattering theory. SIAM, 2013.

[2] Björn Engquist, Anna-Karin Tornberg, and Richard Tsai. Discretization of dirac delta functions in level set methods. Journal of Computational Physics, 207(1):28-51, 2005.

[3] Gerald B Folland. Introduction to partial differential equations. Princeton university press, 1995.

[4] Rainer Kress. Linear integral equations, volume 82 of applied mathematical sciences, 1999.

[5] Catherine Kublik, Nicolay M Tanushev, and Richard Tsai. An implicit interface boundary integral method for poisson?s equation on arbitrary domains. Journal of Computational Physics, 247:279-311, 2013.

[6] Stanley Richardson. Hele shaw flows with a free boundary produced by the injection of fluid into a narrow channel. Journal of Fluid Mechanics, 56(4):609-618, 1972.

[7] P.G. Saffman and F.R.S. Sir Geoffrey Taylor. The penetration of a fluid into a porous medium or hele-shaw cell containing a more viscous liquid. pages $155-174,1988$.

[8] Alok Sutradhar, Glaucio Paulino, and Leonard J Gray. Symmetric Galerkin boundary element method. Springer Science \& Business Media, 2008.

[9] Hongkai Zhao. A fast sweeping method for eikonal equations. Mathematics of computation, 74(250):603-627, 2005. 
